\title{
Subnetwork identification and chemical modulation for neural regeneration: A study combining network guided forest and heat diffusion model
}

\author{
Hui Wang ${ }^{1, \dagger}$, Gang Wang ${ }^{1,2,3, \dagger}$, Li-Da Zhu ${ }^{1}$, Xuan $\mathrm{Xu}^{1}$, Bo Diao ${ }^{2,3, *}$ and Hong-Yu Zhang ${ }^{1, *}$ \\ ${ }^{1}$ Hubei Key Laboratory of Agricultural Bioinformatics, College of Informatics, Huazhong Agricultural University, Wuhan 430070, \\ China \\ ${ }^{2}$ Department of Clinical Experiment, Wuhan General Hospital of Guangzhou Command, Wuhan 430070, China \\ ${ }^{3}$ Hubei Key Laboratory of Central Nervous System Tumor and Intervention, Wuhan 430070, China \\ * Correspondence: dpitao@163.com, zhy630@mail.hzau.edu.cn
}

Received June 10, 2018; Revised July 15, 2018; Accepted August 2, 2018

\begin{abstract}
Background: The induction of neural regeneration is vital to the repair of spinal cord injury (SCI). While compared with peripheral nervous system (PNS), the regenerative capacity of the central nervous system (CNS) is extremely limited. This indicates that modulating the molecular pathways underlying PNS repair may lead to the discovery of potential treatment for CNS injury.

Methods: Based on the gene expression profiles of dorsal root ganglion (DRG) after a sciatic nerve injury, we utilized network guided forest (NGF) to rank genes in terms of their capacity of distinguishing injured DRG from shamoperated controls. Gene importance scores deriving from NGF were used as initial heat in a heat diffusion model (HotNet2) to infer the subnetworks underlying neural regeneration in the DRG. After potential regulators of the subnetworks were found through Connectivity Map (cMap), candidate compounds were experimentally evaluated for their capacity to regenerate the damaged neurons.

Results: Gene ontology analysis of the subnetworks revealed ubiquinone biosynthetic process is crucial for neural regeneration. Moreover, almost half of the genes in these subnetworks are found to be related to neural regeneration via text mining. After screening compounds that are likely to modulate gene expressions of the subnetworks, three compounds were selected for the experiment. Of them, trichostatin A, a histone deacetylase inhibitor, was validated to enhance neurite outgrowth in vivo via an optic nerve crush mouse model.

Conclusions: Our study identified subnetworks underlying neural regeneration, and validated a compound can promote neurite outgrowth by modulating these subnetworks. This work also suggests an alternative approach for drug repositioning that can be easily extended to other disease phenotypes.
\end{abstract}

Keywords: network guided forest; HotNet2; neural regeneration; axon growth; neurotrophic factors

Author summary: Compared with peripheral nervous system, the regenerative capacity of the central nervous system is extremely limited. Thus, it may lead to the discovery of treatment for central nerve injury by modulating gene networks underlying peripheral nerve injury. Based on the gene expression profiles of dorsal root ganglion after a sciatic nerve injury, we defined gene networks underlying peripheral nerve injury. After screening compounds that were likely to modulate gene expressions of the networks, trichostatin A, a histone deacetylase inhibitor, was validated to enhance neurite outgrowth in vivo via an optic nerve crush mouse model.

${ }^{\dagger}$ These authors contributed equally to this work. 


\section{INTRODUCTION}

Treatment for spinal cord injury (SCI) has been focused on stabilizing the spine and controlling inflammation to prevent further injury [1]. SCI have always been considered to be difficult to treat due to its extremely limited capacity of regeneration. On the other hand, peripheral nervous system (PNS) has the ability to switch from arborizing to elongating process after a conditioning lesion [2]. Compared with PNS, central nervous system's (CNS) inability to regenerate itself can be ascribed to the following reasons: first, its regeneration is suppressed by extrinsic inhibitory factors [3-7]; second, CNS lacks the intrinsic capacity of initiating the regeneration process $[8,9]$. In CNS, there are multiple inhibitory factors preventing nerve from regenerate. Astrocytes can produce an impermeable gliotic scar, which act as a barrier for axon outgrowth [3]. The contribution of scar formation in the suppression of axonal growth has been tested by using chondroitinase to increase the permissiveness of injured spinal cord sections to embryonic dorsal root ganglion (DRG) axons [10]. Another inhibitory factor is CNS myelin, which include myelin-associated glycoprotein (MAG) [6], neurite outgrowth inhibitor-A (Nogo-A) [11] and oligodendrocyte myelin glycoprotein (OMgp) [7] . These myelin inhibitors have been proved by the use of monoclonal antibodies IN-1 that can neutralize their inhibitory effect $[11,12]$. In PNS, the complex molecular signals retrogradely transported from axonal lesion to cell body induce its intrinsic growth capacity [13]. In particular, a bunch of mitogen-activated protein kinases (MAPKs) are retrogradely transported, which have been confirmed to participate in the regeneration and repair of neurons [14]. The retrograde transport of injury signals, induces the expression of regeneration-associated genes (RAGs) such as Atf3, Jun and Gap43 [8]. The importance of intrinsic growth capacity in PNS is highlighted by the little axon regeneration observed even after the removal of known inhibitory factors [15].

To address the challenges faced by spinal cord injuries, several approaches have already been proposed. The most commonly accepted method is cellular replacement. For instance, it can be used in the case of the loss of motor neurons resulting from the cervical spine injury [16]. Cellular replacement can be achieved by either transplantation of neural stem cells or in situ stimulation of uninjured stem cells. Besides, some growth-promoting molecules such as neurotransmitter (Acelylcholine), growth factors (GFG, IGF, PDGF), neurotrophic factors (NGF, BDNF, CNTF, NT-3, GDNF), and immunoglobins (N-CAM, L1, cadherins), can be used in axon guidance, fasciculation, and synapse formation [17-20]. Gene therapy and immunization that specifically target different processes of neural regeneration, such as neurotrophic factor delivery, removal of growth inhibition, manipulation of intracellular signaling, and modulation of immune response, have also been developed [21-23]. However, it is very important to take into consideration the inherent plasticity and adaptiveness when a regeneration experiment is interpreted. And the above-mentioned approaches are still far from perfect solutions to the problem of functional regeneration.

Recently, it has been reported that neural repair requires the coordinated transcriptional regulation of a list of core gene modules. In addition, chemical modulation of this list of modules led to the discovery of effective compounds that promoted neural regeneration [24]. Based on this premise, we attempted to find subnetworks that represent perturbed pathways in neural regeneration by systems biology analysis of dorsal root ganglia gene expressions and protein-protein associations. As is shown in the workflow (Figure 1), expression profiles of the dorsal root ganglion were divided into case and control groups in terms of whether the animal suffered from a sciatic injury. By using gene expression profiles, networkguided forest (NGF) assigned importance scores to each gene node in the forest of trees according to a reduction of Gini index at each split $[25,26]$. Then, HotNet2 was used to group genes into functional subnetworks through a heat diffusion process based on protein-protein associations network [27], where the importance scores deriving from NGF served as initial heat inputs. Finally, Connectivity Map (cMap) [28], guided the selection of small molecules that might regulate the gene expression of the core subnetworks. After the experimental validation of three candidate compounds, only trichostatin A was found to significantly promote axon regrowth after an optic nerve injury.

\section{RESULTS}

\section{Importance genes in neural regeneration were identified by network guided forest}

Neural regeneration-associated pathways were wellrepresented in the top genes defined by NGF. Commonly, differentially expressed genes (DEGs) are used to prioritize genes pertaining to a specific state. However, DEGs are notorious for a high false positive rate, which are often ascribed to batch effect and many other confounding factors [29]. Since NGF integrates proteinprotein associations into the random forest to accurately discriminate the injured neurons from sham-operated controls, it leads to a much more precise and robust gene prioritization. Using 5-fold cross-validation, NGF achieved a good performance, with an accuracy of 0.83 and the area under the receiver operating characteristic (ROC) curve (AUC) of 0.90 (Figure 2A). Genes were 


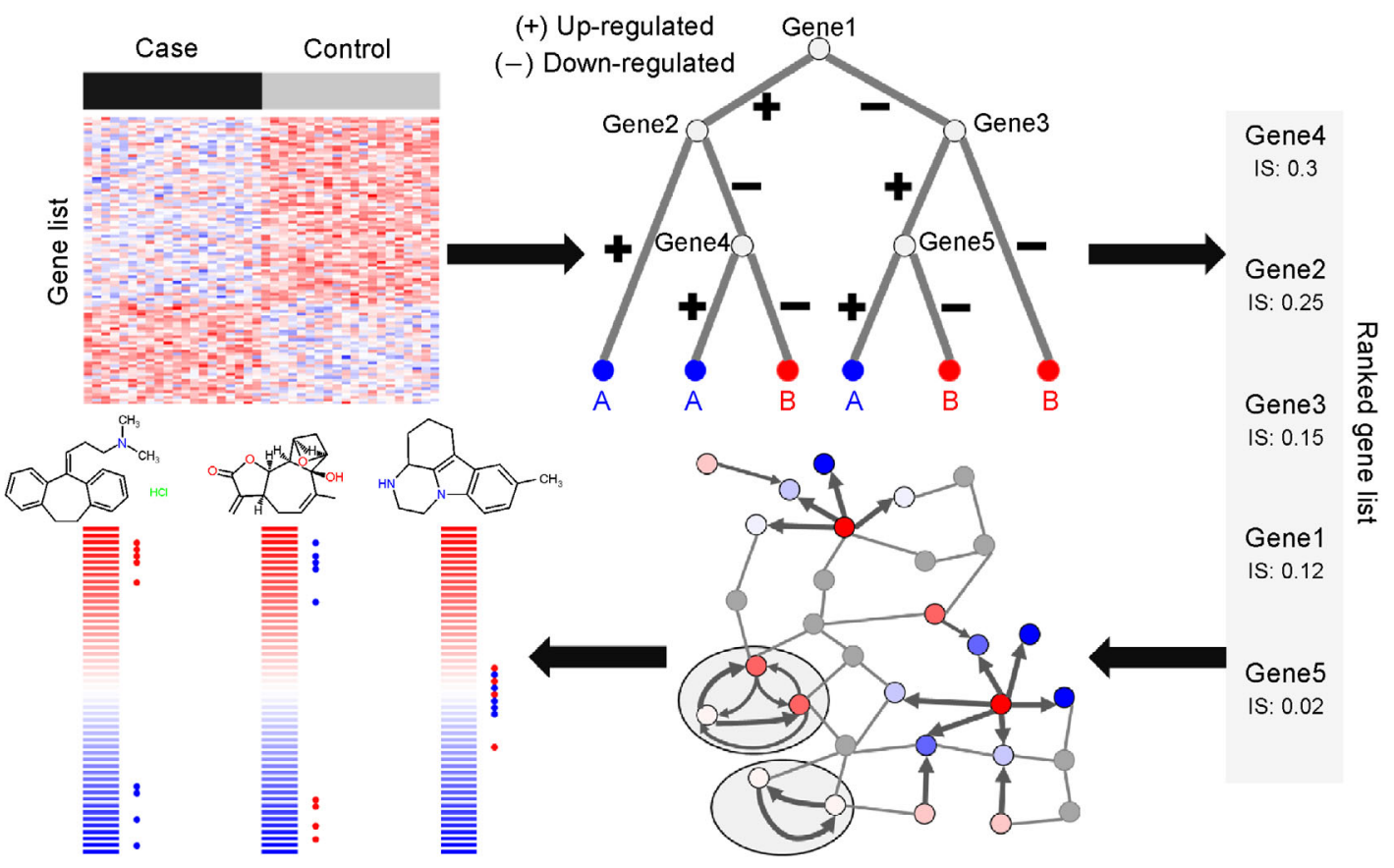

Figure 1. The workflow of our drug screening process. Expression profile of genes were used by network guided forest (NGF) to obtain gene importance scores (Top); Use importance scores from NGF as initial heat scores in HotNet2 to find closely connected subnetworks (Bottom right); The similarity between the query signature (HotNet2 subnetworks) and more than 7,000 expression profiles for 1,309 small molecules was evaluated to find potential compound that might modulate the expression of subnetworks (Bottom left).

Table 1. Classification accuracy of NGF and RF (Number of trees is 1000)

\begin{tabular}{lcc}
\hline Model & AUC & ACCURACY \\
\hline Network-guided forest & $0.892 \pm 0.009$ & $0.821 \pm 0.0147$ \\
Random forest & $0.875 \pm 0.168$ & $0.814 \pm 0.0253$ \\
\hline
\end{tabular}

ranked according to its contribution to classification accuracy (Figure 2B). It is worth noting that the top 100 genes ranked by NGF still tend to be DEGs but also cover genes that do not display significant variability between cases and controls (Figure 2C). Many top genes ranked by NGF but not identified by DEGs are related to neural regeneration (Supplementary Materials 1). In particular, key factors of neural regeneration, such as $B d n f$ and Plcb1, are among the top genes ranked by NGF but absent in the top genes defined by fold changes (Figure 2C). Moreover, gene ontologies of the top 100 scoring genes consist of relevant terms, such as MAPK cascade, oxidative stress, programmed cell death, cell cycle and response to wounding (Supplementary Materials 2). In comparison, gene ontologies of the top 100 genes ranked by fold change $(50$ most upregulated and 50 most downregulated genes) hardly contain any neurodegeneration-related terms except for cAMP signaling (Supplementary Materials 2). Overall, NGF-discovered top genes surpass DEGs by their broad coverage, ranging from the immediate immune response after neurite damage to the regrowth and regeneration capacity that follows the injury. And compared with traditional random forest, NGF not only got a slightly better classification accuracy (Table 1) but also discovered more biologically relevant genes related to neural regeneration. Specifically, 41 of top 100 scored genes by NGF are related to neural regeneration via searching key words "neuronal regeneration", "axonal regeneration", and "nerve injury" in PubMed database, while only 28 of top 100 scored genes by traditional random forest are related to it (Supplementary Materials 1).

\section{Closely connected subnetworks obtained by HotNet2}

To find subnetworks underlying neural regeneration, importance scores of genes obtained via NGF were used as initial heat scores in the heat diffusion model (Figure 1). Six significant subnetworks corresponding to 65 genes were identified (Figure 3). To functionally annotate the subnetworks, we examined the association with the key words "neuronal regeneration", "axonal regeneration", and "nerve injury" in PubMed database for 
A
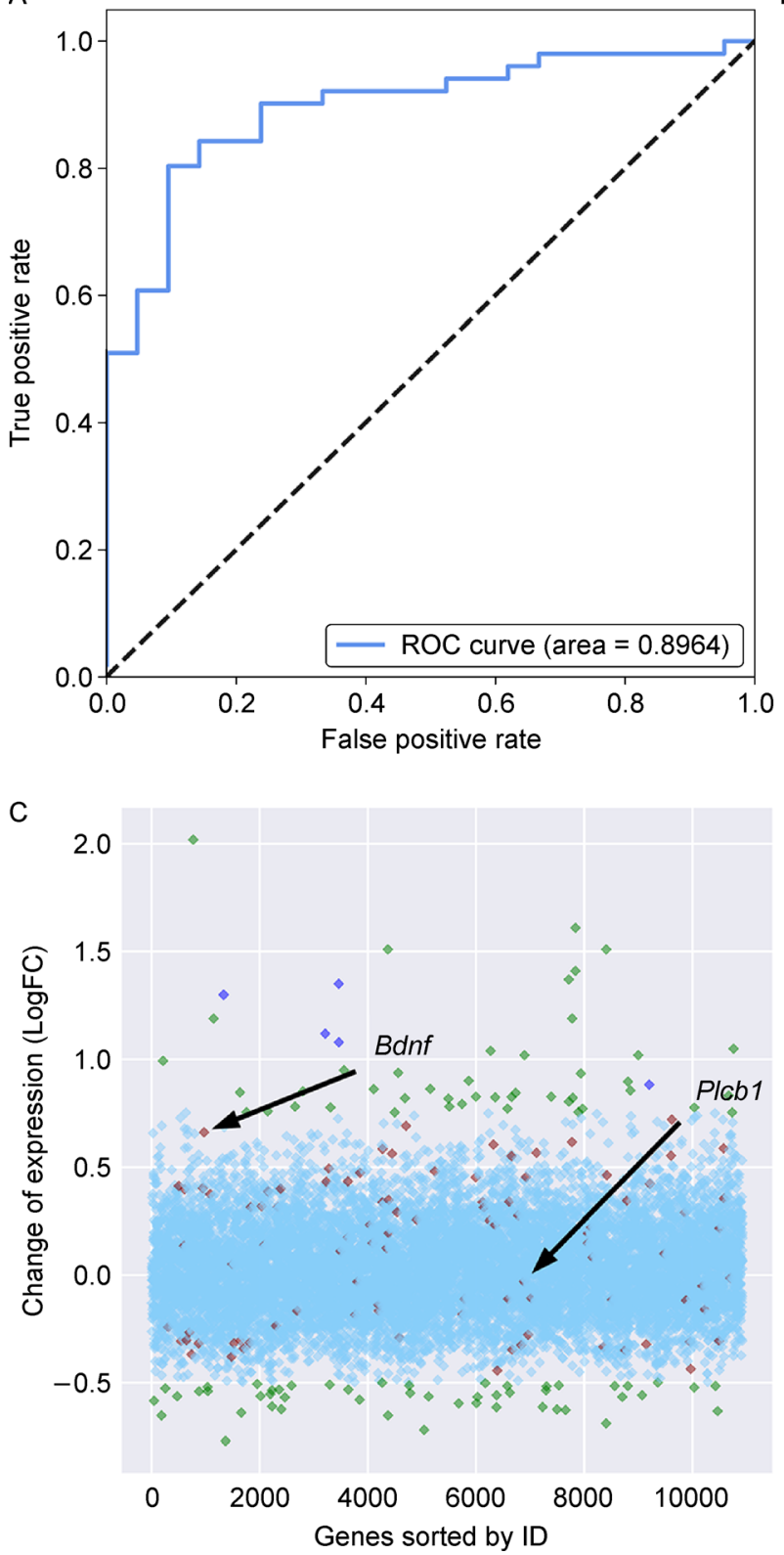

B
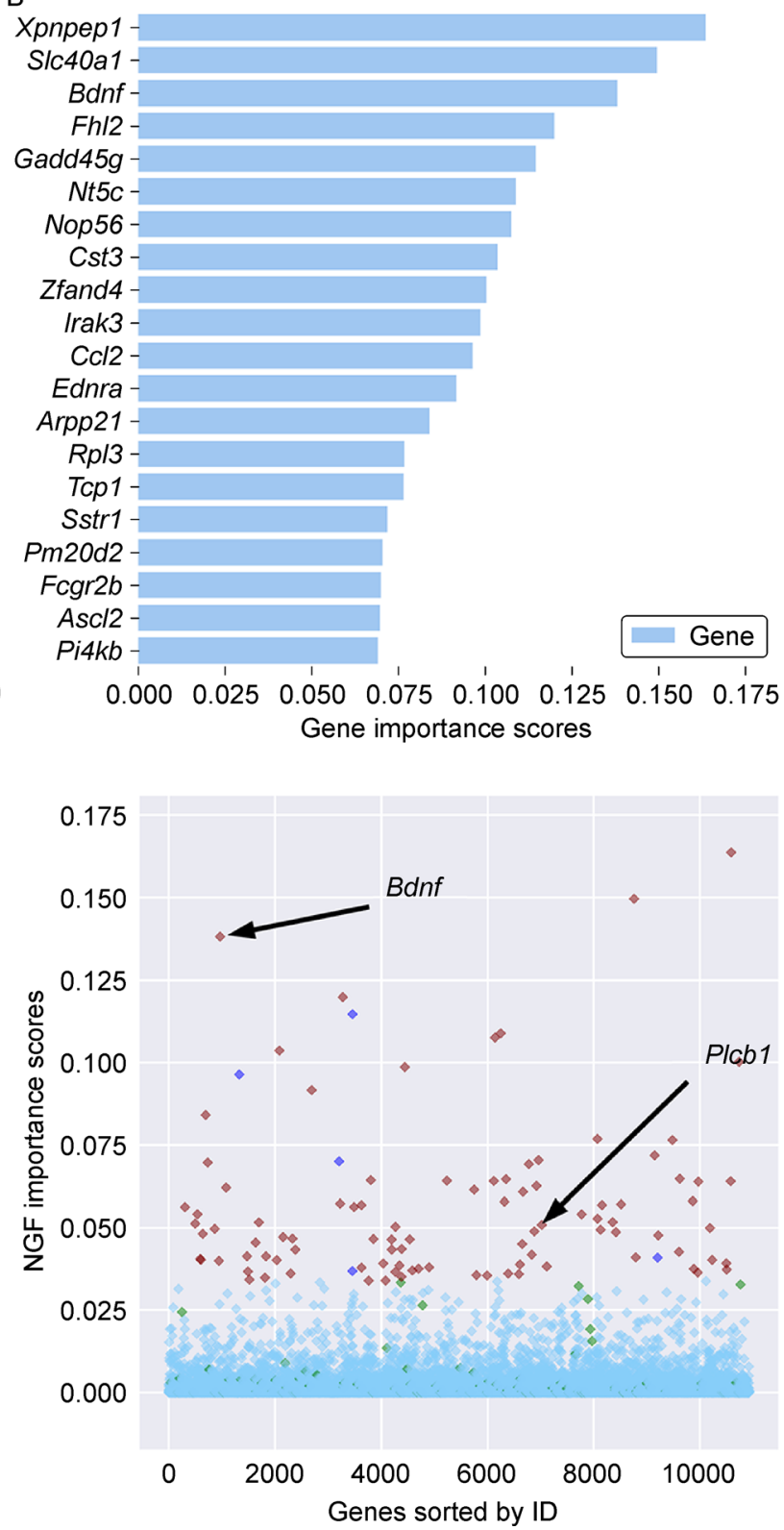

Figure 2. Area under ROC and gene importance scores obtained by NGF. (A) NGF $(10,000$ trees) shows a high AUC of 0.8964. (B) Top 20 genes ranked by NGF. Gene importance scores given by NGF are represented on $x$-axis, and gene names ordered by their importance are shown on $y$-axis. (C) Left: differentially expressed genes (DEGs) by fold change; Right: NGF gene importance scores. The top 100 DEGs (green) and the top 100 genes with the highest NGF importance scores (maroon) are shown. The genes that are in both top lists are shown as blue. Other genes are shown as light sky blue. Overall, the top 100 genes ranked by NGF tend to be differentially expressed but also consist of genes with little variability. Bdnf and Plcb1 are pointed out, as they are important genes related to neuronal regeneration, and they get much higher rank by NGF.

each gene. Nearly $35 \%$ of the genes (24 of 65 genes; hypergeometric $P$ value $3.5 \times 10^{-5}$ ) were associated with neural regeneration and/or axon outgrowth (Supplementary Table S2). Furthermore, using a text mining toolkit, DiGSeE [30], 29 of 65 genes were related to neurodegenerative disease, corresponding to 817 documents (Figure 3).
It is worth noting that transthyretin (TTR), which has the highest degree in one subnetwork, is also the most important node according to the number of associated publications recorded in DiGSeE. TTR is a carrier protein that transports thyroid hormones in the plasma and cerebrospinal fluid that can directly regulate neural regeneration. After nerve crush, knockout mice of Ttr 


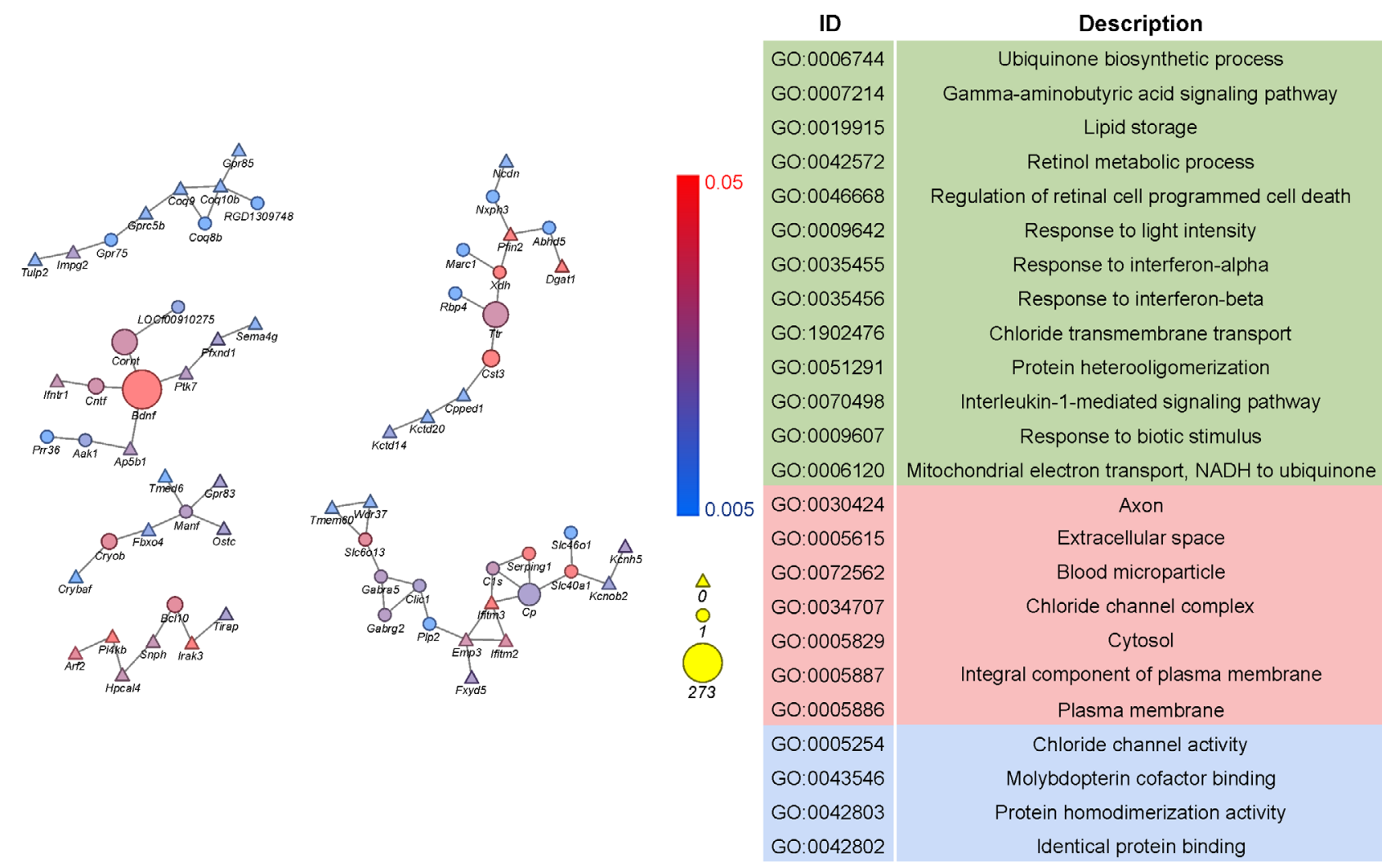

\begin{abstract}
Figure 3. Six subnetworks identified by Hotnet2 and their enriched gene ontology (GO) terms. (A) HotNet2 subnetworks: Colors represent the heat values assigned by HotNet2; Circles represent nodes with literature support, while triangles represent nodes without any literature support; Size of the circles indicates the number of publications that support the associations between the node gene and neurodegenerative diseases. (B) GO terms of subnetwork genes with a $P$ value $<0.05$. Green indicates biological processes, red indicates cellular components, blue indicates molecule functions. As shown, the most enriched cellular component is the axon. And relevant biological processes such as response to interferon and the ubiquinone biosynthetic process are also enriched. Other enriched terms include the gamma-aminobutyric acid signaling pathway, lipid storage, the retinol metabolic process, the regulation of retinal cell programmed cell death, response to light intensity, etc.
\end{abstract}

presented delayed functional recovery resulting from decreased number of myelinated and unmyelinated fibers, while expression of human TTR in neurons rescued this phenotype [31]. Another subnetwork is also of great interest because it contains the associations of BDNF and CNTF and of BDNF and COMT. One polymorphism $\left(\mathrm{Val}^{108} /{ }^{158} \mathrm{Met}\right)$ of COMT accounts for a 4-fold variation in enzyme activity and dopamine catabolism [32]. And consistent with the interaction in the subnetwork, COMT and BDNF have been found to interactively influence the working memory [33]. BDNF and CNTF are two neurotrophic factors that can support the survival of existing neurons and enhance neurite outgrowth. Conforming with the interaction in the subnetwork, combined treatment of BDNF and CNTF increases the rate of nerve regeneration over treatment with BDNF alone [34].

\section{Transcription factor binding site enrichment and in silico drug screeen for neural regeneration}

AP-1 and Pax 2 are enriched as the network regulators via transcription factor binding site enrichment analysis using a web-based tool named Enrichr [35]. Pax2 are expressed in populations of nerve cells within the developing forebrain, midbrain, hindbrain and spinal cord [36,37]. During CNS development, Pax2 have substantial roles in neural differentiation and regional specification, which also indicates its potential implications in neural regeneration $[36,38]$. More importantly, AP-1 has been widely recognized as a major effector in neural regeneration. AP-1 transcription factor c-Jun is highly expressed in response to neuronal trauma. Its absence can cause severe defects in perineuronal sprouting, lymphocyte recruitment and microglial activation, and its over-expression 
significantly increases neurite outgrowth [24,39]. The enrichment of AP-1 and Pax2 not only prove that subnetworks that we discovered are promising targets for neuronal regeneration but also indicates the potential in regulation of these six subnetworks to the discovery of small molecules for neuronal regeneration.

To identify small molecules that can modulate genes in subnetworks, cMap, which consists of reference expression profiles for 1309 small molecules, was employed. cMap allow users to upload their list of down-regulated genes and up-regulated genes as a query signature to find small molecules that display similar or converse effects [40]. Based on a comparison of the expression profiles of these 65 subnetwork genes with the 1309 reference profiles in the cMap database, four compounds with most significant permuted $P$ values were chosen (Supplementary Materials 3). Among these compounds, rottlerin, a protein kinase $\mathrm{C}$ delta specific inhibitor has already been found to affect neurite outgrowth in dorsal root ganglion [41]. The other three compounds (trichostatin A, pirlindole and amitriptyline) were chosen for further experimental evaluation. Moreover, we also query the signature against L1000 array, which contains over $1 \mathrm{M}$ gene expression profiles [42]. Interestingly, only trichostatin $\mathrm{A}$ are in top 50 identified drugs by $\mathrm{L} 1000$ (Supplementary Materials 3), which is consistent with our experimental validation of drug efficacy.

\section{In vivo experimental validation}

It has been reported that differences in the regenerative potential between injured PNS neurons and injured CNS neurons likely reflect major differences in intrinsic transcriptional networks [24]. Thus, we tested candidate drugs on their effects on optic nerve regeneration which is a standard model for CNS regeneration [43]. Uninjured and untreated mice were used as a reference. Experimental mice were suffered from an optic nerve crush injury and divided into five groups: mice treated with ambroxol (ambroxol has been proven to be able to promote CNS regeneration) [24] as the positive control group; mice treated with trichostatin A, pirlindole and amitriptyline as the main experimental groups; mice treated with vehicle as the negative control group; after two weeks, we examined the optic nerve regeneration via hematoxylin-eosin (HE) staining and immunofluorescence staining. From HE staining image, uninjured optic nerve showed neatly arranged and homogeneously dyed axon fibers, and uniformly distributed glial cells; while the optic nerve from the injured but untreated mice showed sparse and lightly stained axon fibers, lost of glial cells with vacuolar degeneration and necrosis. Although no improvement was observed in amitriptyline or pirlindole treated group, the axon fibers and glial cells of optic nerve were recovered in trichostatin A treated group (Figure 4). Further immunofluorescence showed, among the three tested compounds, trichostatin A displayed a significant increase in axon regeneration beyond the site of the lesion (Figure 5A and $5 \mathrm{~B})$. Even though pirlindole and amitriptyline showed a significantly larger $\beta 3$-tubulin staining area (Student's t-test $P<0.05$ ) at one locus (Figure 5B), the lack of significant larger staining area near crush site indicates these may be false signals. In contrast, trichostatin A significantly promoted axon regeneration, and its $\beta 3$ tubulin staining areas were significantly larger than the negative control at loci at different distances from the crush site (Student's t-test $P<0.05$; Figure 5B). Moreover, the staining area of trichostatin A was not significantly different from that of ambroxol at each locus, which indicates similar efficacy of trichostatin A and ambroxol on neural repair (Figure 5B). It was reported that trichostatin A selectively inhibits the class I and II mammalian histone deacetylase families of enzymes [44]; thus, it can be used to alter gene expression by interfering with the removal of acetyl groups from histones and facilitating its regulation of subnetwork genes. Overall, the ability of trichostatin A to promote neural regeneration is of great interest for the treatment of spinal cord injury or neurodegenerative diseases.

The optic nerve of normal mice showed neatly arranged and homogeneously dyed axon fibers, and uniformly distributed glial cells; while the optic nerve of injured but untreated mice showed sparse and lightly stained axon fibers, lost of glial cells with vacuolar degeneration and necrosis. In ambroxol treated group and trichostatin A treated group, the axon fibers and glial cells of optic nerve were recovered, while no improvement in pirlindole or amitriptyline treated group.

\section{DISCUSSION}

A previous study reported approximately 4500 DEGs after neural injury and identified 400 redundant signaling networks related to 39 transcription factors [13], which revealed the complexity of signaling transduction after neural injury. However, there must be a core set of upstream genes that modulate such complex responses. In the present work, the subnetworks coordinating the complicated signaling responses following neural injury were identified by combining the network guided forest and a heat diffusion model. In these subnetworks, two importance neurotrophic factors, namely, BDNF and CNTF, are vital for the repair and regrowth of injured neurons. And genes in each subnetwork are closely connected to each other. For example, the interaction between COMT and BDNF has been proved to influence working memory [33]. Moreover, almost half of the genes in the subnetworks are related to neural regeneration or 


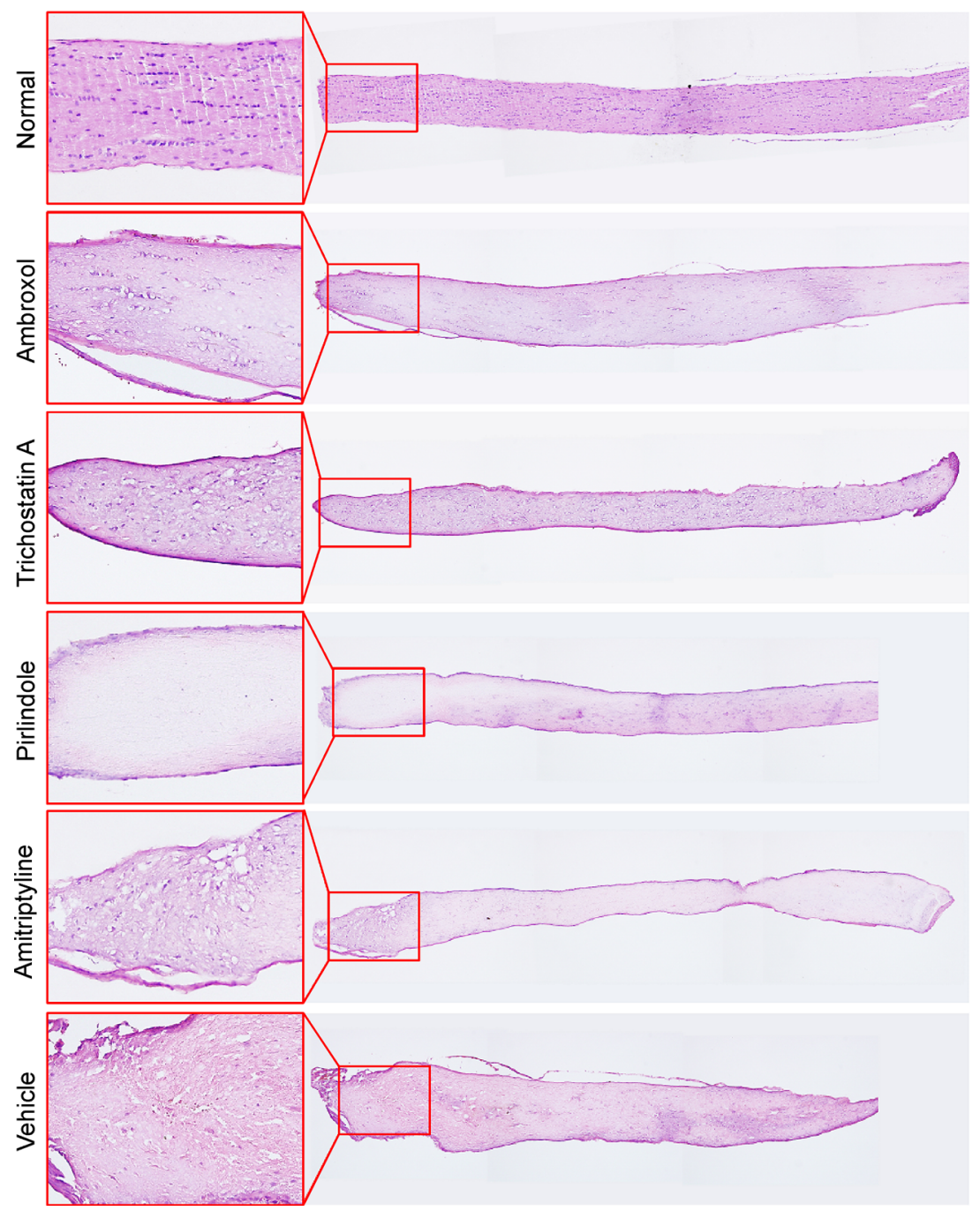

Figure 4. HE staining of optic nerve in mice. The optic nerve of normal mice showed neatly arranged and homogeneously dyed axon fibers, and uniformly distributed glial cells; while the optic nerve of injured but untreated mice showed sparse and lightly stained axon fibers, lost of glial cells with vacuolar degeneration and necrosis. In ambroxol treated group and trichostatin A treated group, the axon fibers and glial cells of optic nerve were recovered, while no improvement in pirlindole or amitriptyline treated group.

neural injury. Conventional drug-targeted mechanisms such as immune response and neurotrophic factors, are also well captured by subnetworks. Therefore, the other half of genes in the subnetworks are potential regulators in the neural regeneration. Further validation of these subnetwork genes may lead to the new discovery of the underlying mechanisms of neural regeneration.

By screening compounds that are likely to modulate the gene expressions of subnetworks, we can find compounds that are able to systematically regulate complex signaling pathways underlying neural regeneration. Based on this principle, we utilized cMap, a systematic pharmacological approach, to help locate potential network regulators. Of the three candidate compounds that went through experimental validation, optic nerves treated by two compounds displayed slightly higher but not statistically significant immunofluorescence staining area than in vehicle treated group. Only trichostatin A treated group displayed significant neurite regrowth after injury. Compared with traditional high-throughput screening which searches a large chemical space without prior knowledge [38], our approach depends on the core subnetworks we constructed on gene expressions and protein-protein associations. Thus, this approach requires a smaller number of compounds to be tested, and has a higher potential in finding effective drugs.

\section{CONCLUSIONS}

This study demonstrates the application of network 


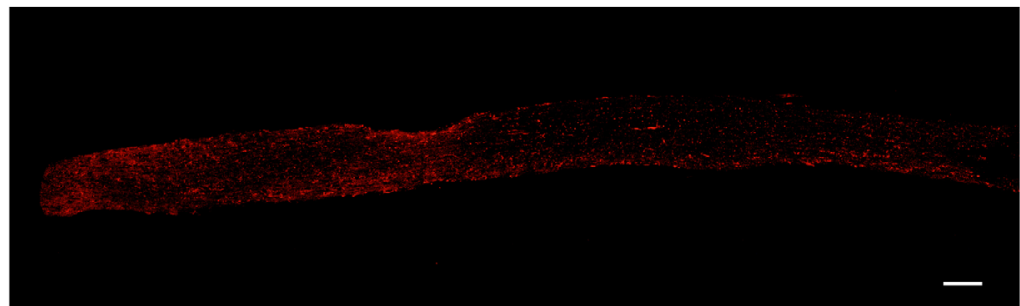

$\varangle$

衰

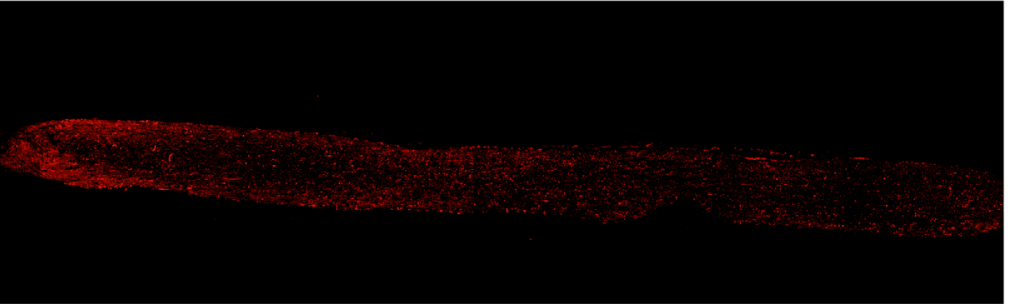

$\frac{\text { 음 }}{\frac{0}{0}}$
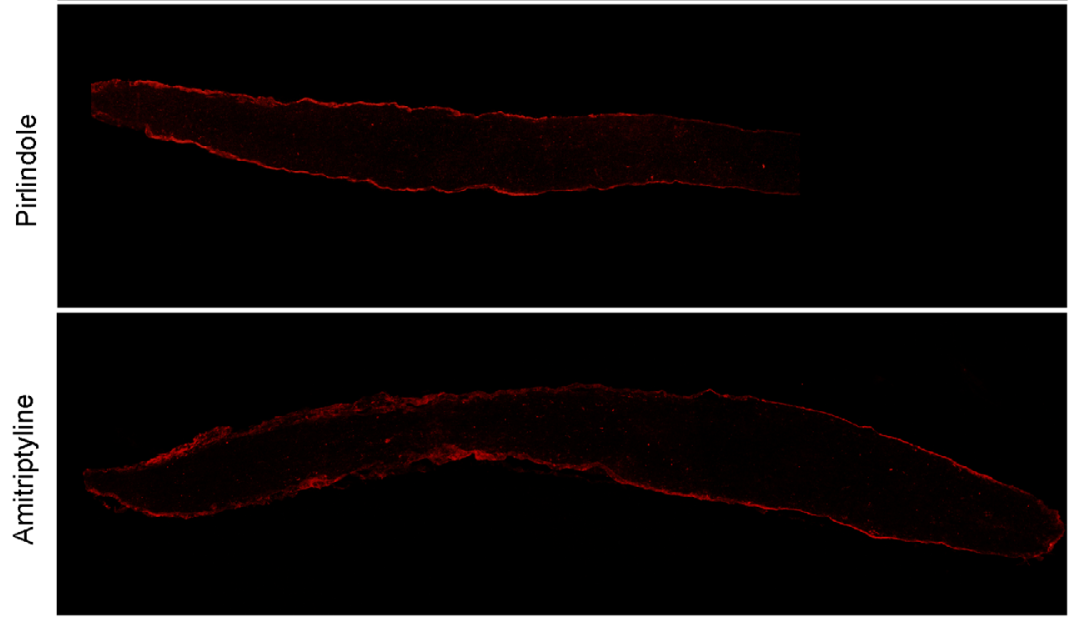

$\frac{\frac{0}{0}}{\frac{0}{\sigma}}$

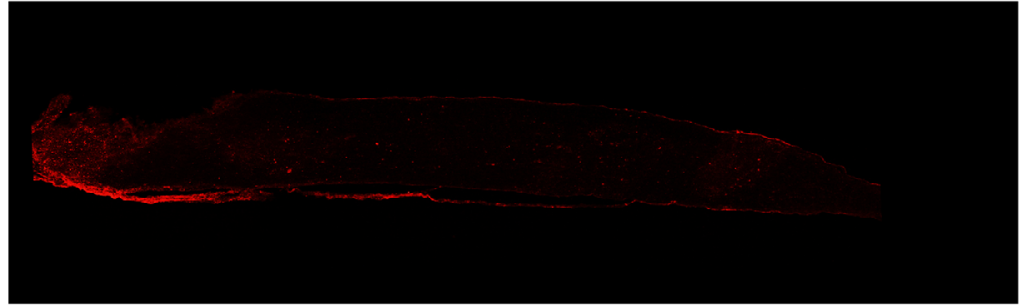

B

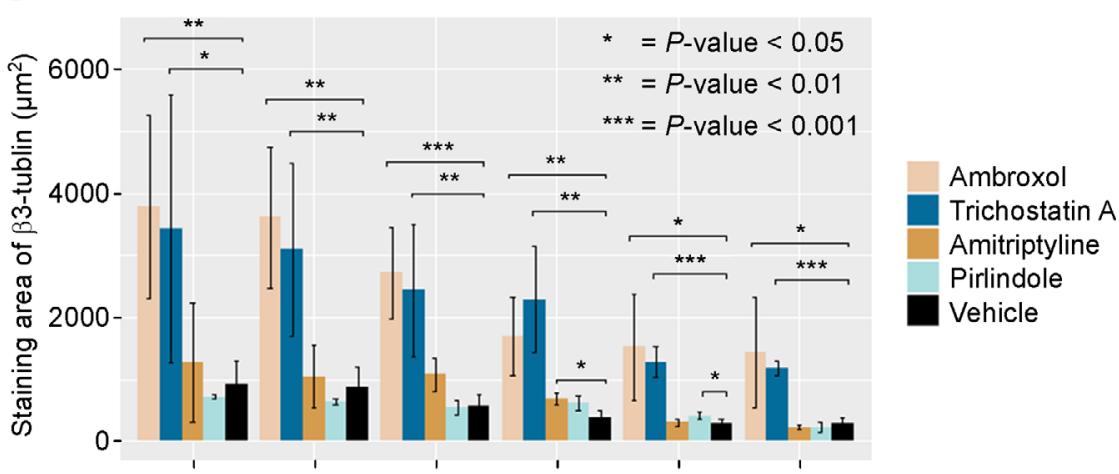

$100-200 \mu \mathrm{m} \quad 400-500 \mu \mathrm{m} \quad 900-1000 \mu \mathrm{m} 1400-1500 \mu \mathrm{m} 1900-2000 \mu \mathrm{m} \quad 2400-2500 \mu \mathrm{m}$

Distance from crush site

Figure 5. Immunofluorescence staining of optic nerve regeneration. (A) Axon immunofluorescence staining by $\beta$-III-tubulin. There are 5 experimental groups: at the top of the picture, a nerve treated with ambroxol served as a positive control; in the middle of the picture, nerves respectively treated with three experimental drugs, trichostatin A, pirlindole, and amitriptyline are shown; at the bottom of the picture, a nerve treated with vehicle served as a negative control. Trichostatin $A$ treatment has a similar effect to the positive control, both of which significantly enhanced neurite outgrowth after injury. While pirlindole and amitriptyline did not display significant neurite outgrowth after injury (scale bar, $200 \mu \mathrm{m}$ ). (B) Staining areas of immunofluorescence at different distance from injury. Trichostatin A displayed similar effect in neural regeneration as ambroxol (positive control). While amitriptyline only showed a significant effect at 1400-1500 $\mu \mathrm{m}$, and pirlindole only showed a significant effect at 1900-2000 $\mu \mathrm{m}$. 
pharmacology to the repositioning of drugs for neural regeneration. We prioritize the important genes based on NGF which can integrate gene expressions and proteinprotein associations into one framework. And we find closely connected subnetworks whose interactions underlie the complex signaling responses after neural injury. More importantly, we validated that trichostatin A, a histone deacetylase inhibitor, could promote axon regeneration through modulation of the subnetworks via an optic nerve crush mouse model.

\section{MATERIALS AND METHODS}

\section{Data preparation: gene expression profiles and protein-protein associations}

Expression data can be downloaded from GEO database using accession number GSE26350 [13]. L4/L5 dorsal root ganglia were dissected from crush-lesioned or control sham-operated rats at different time points. mRNA expressions were quantified via rat genome 230.2 arrays (Affymetrix). Data were then pre-processed with the RMA algorithm [45] and log2-transformed.

Protein-protein associations were downloaded from the STRING database [46]. Apart from available experimental data on protein-protein interactions, and importing known pathways and protein complexes from curated databases, protein-protein associations are derived from the following sources: (i) systematic co-expression analysis, (ii) detection of shared selective signals across genomes, (iii) automated text-mining of the scientific literature and (iv) computational transfer of interaction knowledge between organisms based on gene orthology. And only protein-protein associations with a score $>=$ 400 were kept for further analysis.

\section{Network guided forest (NGF) analysis}

NGF uses gene expression profiles and protein association networks as inputs to train a random forest classification model that can precisely differentiate a case from a control. In our case, expression profiles were divided into injured DRGs and sham-operated controls. The algorithm is a revised random forest algorithm. The main difference in NGF is that the selection of nodes at each branch must take protein networks into consideration. As in random forest, each tree is constructed based on a bootstrap subset of samples drawn with replacement from original samples. And each tree is built using the recursive partitioning algorithm CART (classification and regression trees) [47]. In NGF, samples for each tree are drawn with replacement from the original sample to form a new sample that has the same size. Second, randomly select $\sqrt{N}$ genes from all the $N$ genes as candidate split nodes for the root, and to put more restriction on root node, only using genes with a degree of at least 5 as root candidates. Third, Gini index were used as a measure of impurity to decide how well a gene and a corresponding expression threshold can differentiate samples with respect to their phenotypic classes; the best such gene is the final chosen node. Fourth, at each subsequent node, the probability of selecting a particular gene for the candidate set is proportional to the number of proteinprotein associations it shares with genes already in the tree; fifth, repeat the third step and fourth step iteratively, to choose the node that bring the largest Gini index reduction at each branch, until the Gini index reduction is smaller than 0.02 . The above steps were repeated 10,000 times, and a total of 10,000 different decision trees were built to form the NGF model related to neural regeneration. According to the reduction of the Gini index at each node in each tree, NGF assigns an importance score for each gene (important score, IS). More specifically, according to the reduction in the output sample Gini index due to the node (gene) split in the decision tree, the key regulation genes and their importance scores (IS) can be obtained:

$$
\begin{gathered}
I S(g)=\operatorname{Gini}(T)-\frac{n_{U}}{N_{T}} \operatorname{Gini}(U)-\frac{n_{D}}{N_{T}} \operatorname{Gini}(D) \\
F=\operatorname{Gini}(S)=1-\left(\frac{n_{P}}{N_{S}}\right)^{2}-\left(\frac{n_{N}}{N_{S}}\right)^{2}
\end{gathered}
$$

where $\operatorname{Gini}(T)$ is the Gini index of all of the samples as tested by split node $g, \operatorname{Gini}(U)$ is the Gini index of the sample subset whose expression values are larger than threshold, and $\operatorname{Gini}(D)$ is the Gini index of the sample subset whose expression values are lower than threshold. $N_{T}, n_{U}$ and $n_{D}$ are the sizes of corresponding sample sets. The Gini index is defined in Equation (2), in which $n_{P}$ and $n_{N}$ represent the number of positive and negative samples, respectively, in the sample set $N_{S}[25,26]$.

\section{HotNet2 analysis}

HotNet2 requires two inputs, one is a heat vector $\vec{h}$, which consists of the initial heat for each gene, another is a network graph $G=(V, E)$, where each node corresponds to a gene and each edge corresponds to an association between two nodes. HotNet2 first captures the local topology of the protein-protein association network around a gene by an insulated heat diffusion process [48]. At each time interval, an insulating parameter $\beta$, controls how much heat a node retains. And each node passes down the rest of heat through protein-protein 
association networks. At equilibrium, the amount of heat on each node is determined by its initial heat, the local topology of the network surrounding the node, and the insulating parameter $\beta$. Assuming each node contains a unit heat, then the heat diffuses from node $i$ to node $j$ is the $(i, j)$ entry of the diffusion matrix $F$ defined by:

$$
F=\beta(I-(1-\beta) W)^{-1},
$$

where

$$
W_{i j}=\frac{1}{\operatorname{deg}(j)},
$$

if node $i$ interacts with node $j, 0$ otherwise. Therefore, the network graph is normalized to get the adjacency matrix $W$. Combining the diffusion matrix with the initial heat of each node, the exchanged heat matrix $E$ is defined as follow:

$$
E=F D_{h},
$$

where $D_{\vec{h}}$ is the diagonal matrix with entries $\vec{h}$. The $(i, j)$ entry of the exchanged heat matrix $E$ is the heat that diffuses from node $g_{j}$ to node $g_{i}$ considering the initial heat on node $j$. If $E(i, j)$ is bigger than a threshold $\delta$, then node $j$ to node $i$ can be connected by a directed edge of weight $E(i, j)$. Then, HotNet2 identifies strongly connected components in a graph through a two-step statistical test $[27,49,50]$. The source code for HotNet2 is freely available at https://github.com/raphael-group/ hotnet 2 .

\section{Functional annotation and enrichment analysis}

To functionally annotate genes in subnetworks, we first search the PubMed database for association with key words, neuronal regeneration, axonal regeneration, and nerve injury for every gene using a web scraping script. Next, employing a text mining toolkit, DiGSeE at https:// gcancer.org/digsee [30], we were able to extract the sentences containing the associations between neural degenerative diseases with each gene. Gene ontology analysis were performed using DAVID functionally analysis toolkit at https://david.ncifcrf.gov [51,52] and Panther at http://www.pantherdb.org [53]. Transcription factor binding sites enrichment analysis was performed using Enrichr at http://amp.pharm.mssm.edu/Enrichr [35].

\section{In silico small molecule screening}

The Connectivity Map (cMap) database (https://portals. broadinstitute.org/cmap) can be used to screen potential drugs, and the details have been described previously
[28]. Essentially, cMap is a database that contains the expression profiles of more than 7000 instances and 1309 small molecules. Users can submit a list of downregulated genes and a list of up-regulated genes associated with a specific perturbation, and cMap then returns the molecules that have the most similar or most dissimilar signatures when compared with the user's inputs. We used 65 genes identified by HotNet 2 as the query signature for cMap. By choosing the molecules with the smallest permutation $P$-value, the top three enriched small molecules in the returned signature lists were utilized for experimental validation.

\section{Mice optic nerve injury and drug administration}

All animals received humane care according to the criteria outlined in the "Guide for the Care and Use of Laboratory Animals" prepared by the National Academy of Sciences and published by the National Institutes of Health (NIH publication 86-23, revised 1985). All of the in vivo experiments comply with the animal study protocol approved by the ethics committee of Wuhan General Hospital of PLA. First six-week-old C57BL/6 mice were obtained and acclimated for one week. Then, the mice were randomly divided into six groups: one group was uninjured and five groups' optic nerves were crushed as described previously [54]. Immediately following injury, the five treatment groups received a $1 \mu \mathrm{L}$ intravitreal injection of either vehicle (1\% DMSO, $5 \%$ Tween- 80 and 5\% PEG 400 in water), Ambroxol $(25 \mathrm{mg} / \mathrm{mL}$ in vehicle), Trichostatin A ( $50 \mu \mathrm{g} / \mathrm{mL}$ in vehicle), Pirlindole $(10 \mathrm{mg} / \mathrm{mL}$ in vehicle) or Amitriptyline $(1 \mathrm{mg} / \mathrm{mL}$ in vehicle). From the day after the optic nerve was crushed to the day prior to termination, each group of mice received a $120 \mu \mathrm{L}$ intraperitoneally injection of either vehicle, Ambroxol $(25 \mathrm{mg} / \mathrm{mL})$, Trichostatin A $(50 \mu \mathrm{g} / \mathrm{mL})$, Pirlindole $(10 \mathrm{mg} / \mathrm{mL})$ or Amitriptyline (1 $\mathrm{mg} / \mathrm{mL}$ ). Uninjured mice were set as the normal control group.

\section{Tissue preparation, hematoxylin-eosin (HE) staining and immunofluorescence staining}

Animals were given a lethal dose of anesthesia (Chloral hydrate) and perfused intracardially with $4 \%$ paraformaldehyde (PFA) in PBS. After dissection, optic nerves were post fixed overnight at $4^{\circ} \mathrm{C}$ in $4 \%$ PFA, and were cryoprotected in $20 \%$ sucrose/PBS for $48 \mathrm{~h}$ at $4^{\circ} \mathrm{C}$. Optic nerves were embedded in Tissue-Tek and $8 \mu \mathrm{m}$ sections were made using a cryostat. Then the optic nerve sections were washed 3 times in PBS before HE staining and immunofluorescence staining. For immunofluorescence staining, optic nerve sections were incubated for $1 \mathrm{~h}$ at room temperature in blocking solution $(5 \% \mathrm{BSA}, 0.25 \%$ 
Triton X-100 in PBS). Beta III Tubulin antibody (Abcam, $1 / 1000$, ab18207) was diluted in blocking solution and incubated with optic nerve sections overnight at $4^{\circ} \mathrm{C}$. Optic nerve sections were then washed 3 times in PBS. The secondary antibody (Goat anti-rabbit IgG-Cy3, Proteintech) was diluted in PBS and incubated with optic nerve sections for $30 \mathrm{~min}$ at $37^{\circ} \mathrm{C}$. Optic nerve sections were then washed 3 times in PBS, mounted with $50 \%$ glycerol/PBS and photographed under a fluorescence microscope.

\section{SUPPLEMENTARY MATERIALS}

The supplementary materials can be found online with this article at https:// doi.org/10.1007/s40484-018-0159-0.

\section{ACKNOWLEDGEMENTS}

This work was supported by the Fundamental Research Funds for the Central Universities (No. 2662017PY115)

\section{COMPLIANCE WITH ETHICS GUIDELINES}

The authors Hui Wang, Gang Wang, Li-Da Zhu, Xuan Xu, Bo Diao and Hong-Yu Zhang declare that they have no conflict of interests.

All procedures performed in studies involving animals were in accordance with the ethical standards of the institution or practice at which the studies were conducted, and with the 1964 Helsinki declaration and its later amendments or comparable ethical standards.

\section{REFERENCES}

1. Hulsebosch, C. E. (2002) Recent advances in pathophysiology and treatment of spinal cord injury. Adv. Physiol. Educ., 26, 238-255

2. Smith, D. S. and Pate Skene, J. H. (1997) A transcriptiondependent switch controls competence of adult neurons for distinct modes of axon growth. J. Neurosci., 17, 646-658

3. Silver, J. and Miller, J. H. (2004) Regeneration beyond the glial scar. Nat. Rev. Neurosci., 5, 146-156

4. McKerracher, L., David, S., Jackson, D. L., Kottis, V., Dunn, R. J. and Braun, P. E. (1994) Identification of myelin-associated glycoprotein as a major myelin-derived inhibitor of neurite growth. Neuron, 13, 805-811

5. Fitch, M. T. and Silver, J. (2008) CNS injury, glial scars, and inflammation: inhibitory extracellular matrices and regeneration failure. Exp. Neurol., 209, 294-301

6. Filbin, M. T. (2003) Myelin-associated inhibitors of axonal regeneration in the adult mammalian CNS. Nat. Rev. Neurosci., 4, 703-713

7. Wang, K. C., Koprivica, V., Kim, J. A., Sivasankaran, R., Guo, Y., Neve, R. L. and He, Z. (2002) Oligodendrocyte-myelin glycoprotein is a Nogo receptor ligand that inhibits neurite outgrowth. Nature, 417, 941-944

8. Schmitt, A. B., Breuer, S., Liman, J., Buss, A., Schlangen, C., Pech, K., Hol, E. M., Brook, G. A., Noth, J. and Schwaiger, F.-W. (2003) Identification of regeneration-associated genes after central and peripheral nerve injury in the adult rat. BMC Neurosci., 4, 8

9. Giger, R. J., Hollis, E. R. 2nd and Tuszynski, M. H. (2010) Guidance molecules in axon regeneration. Cold Spring Harb. Perspect. Biol., 2, a001867

10. Zuo, J., Neubauer, D., Dyess, K., Ferguson, T. A. and Muir, D. (1998) Degradation of chondroitin sulfate proteoglycan enhances the neurite-promoting potential of spinal cord tissue. Exp. Neurol., 154, 654-662

11. Chen, M. S., Huber, A. B., van der Haar, M. E., Frank, M., Schnell, L., Spillmann, A. A., Christ, F. and Schwab, M. E. (2000) Nogo-A is a myelin-associated neurite outgrowth inhibitor and an antigen for monoclonal antibody IN-1. Nature, 403, 434-439

12. Rubin, B. P., Dusart, I. and Schwab, M. E. (1994) A monoclonal antibody (IN-1) which neutralizes neurite growth inhibitory proteins in the rat CNS recognizes antigens localized in CNS myelin. J. Neurocytol., 23, 209-217

13. Michaelevski, I., Segal-Ruder, Y., Rozenbaum, M., Medzihradszky, K. F., Shalem, O., Coppola, G., Horn-Saban, S., BenYaakov, K., Dagan, S. Y., Rishal, I., et al. (2010) Signaling to transcription networks in the neuronal retrograde injury response. Sci. Signal., 3, ra53

14. Nix, P., Hisamoto, N., Matsumoto, K. and Bastiani, M. (2011) Axon regeneration requires coordinate activation of $\mathrm{p} 38$ and JNK MAPK pathways. Proc. Natl. Acad. Sci. USA, 108, 10738-10743

15. Yiu, G. and He, Z. (2006) Glial inhibition of CNS axon regeneration. Nat. Rev. Neurosci., 7, 617-627

16. Horner, P. J. and Gage, F. H. (2000) Regenerating the damaged central nervous system. Nature, 407, 963-970

17. Mattson, M. P. (1989) Acetylcholine potentiates glutamateinduced neurodegeneration in cultured hippocampal neurons. Brain Res., 497, 402-406

18. Connor, B. and Dragunow, M. (1998) The role of neuronal growth factors in neurodegenerative disorders of the human brain. Brain Res. Rev., 27, 1-39

19. Kamei, N., Tanaka, N., Oishi, Y., Hamasaki, T., Nakanishi, K., Sakai, N. and Ochi, M. (2007) BDNF, NT-3, and NGF released from transplanted neural progenitor cells promote corticospinal axon growth in organotypic cocultures. Spine, 32, 1272-1278

20. Ziegner, U. H., Kobayashi, R. H., Cunningham-Rundles, C., Español, T., Fasth, A., Huttenlocher, A., Krogstad, P., Marthinsen, L., Notarangelo, L. D., Pasic, S., et al. (2002) Progressive neurodegeneration in patients with primary immunodeficiency disease on IVIG treatment. Clin. Immunol., 102, 19-24

21. Blesch, A., Lu, P. and Tuszynski, M. H. (2002) Neurotrophic factors, gene therapy, and neural stem cells for spinal cord repair. Brain Res. Bull., 57, 833-838

22. Huang, D. W., McKerracher, L., Braun, P. E. and David, S. (1999) A therapeutic vaccine approach to stimulate axon regeneration in the adult mammalian spinal cord. Neuron, 24, 639-647

23. Sicotte, M., Tsatas, O., Jeong, S. Y., Cai, C.-Q., He, Z. and David, S. (2003) Immunization with myelin or recombinant Nogo-66/ MAG in alum promotes axon regeneration and sprouting after corticospinal tract lesions in the spinal cord. Mol. Cell. Neurosci., 23, 251-263

24. Chandran, V., Coppola, G., Nawabi, H., Omura, T., Versano, R., 
Huebner, E. A., Zhang, A., Costigan, M., Yekkirala, A., Barrett, L., et al. (2016) A systems-level analysis of the peripheral nerve intrinsic axonal growth program. Neuron, 89, 956-970

25. Dutkowski, J. and Ideker, T. (2011) Protein networks as logic functions in development and cancer. PLoS Comput. Biol., 7, e1002180

26. Dong, X., Jiang, Z., Peng, Y. L. and Zhang, Z. (2015) Revealing shared and distinct gene network organization in Arabidopsis immune responses by integrative analysis. Plant Physiol., 167, $1186-1203$

27. Leiserson, M. D. M., Vandin, F., Wu, H. T., Dobson, J. R., Eldridge, J. V., Thomas, J. L., Papoutsaki, A., Kim, Y., Niu, B., McLellan, M., et al. (2015) Pan-cancer network analysis identifies combinations of rare somatic mutations across pathways and protein complexes. Nat. Genet., 47, 106-114

28. Lamb, J., Crawford, E. D., Peck, D., Modell, J. W., Blat, I. C., Wrobel, M. J., Lerner, J., Brunet, J. P., Subramanian, A., Ross, K. N., et al. (2006) The Connectivity Map: using gene-expression signatures to connect small molecules, genes, and disease. Science, 313, 1929-1935

29. Johnson, W. E., Li, C. and Rabinovic, A. (2007) Adjusting batch effects in microarray expression data using empirical Bayes methods. Biostatistics, 8, 118-127

30. Kim, J., So, S., Lee, H. J., Park, J. C., Kim, J. J. and Lee, H. (2013) DigSee: disease gene search engine with evidence sentences (version cancer). Nucleic Acids Res., 41, W510-W517

31. Fleming, C. E., Saraiva, M. J. and Sousa, M. M. (2007) Transthyretin enhances nerve regeneration. J. Neurochem., 103, 831-839

32. Egan, M. F., Goldberg, T. E., Kolachana, B. S., Callicott, J. H., Mazzanti, C. M., Straub, R. E., Goldman, D. and Weinberger, D. R. (2001) Effect of COMT Val ${ }^{108} /{ }^{158}$ Met genotype on frontal lobe function and risk for schizophrenia. Proc. Natl. Acad. Sci. USA, 98, 6917-6922

33. Chen, W., Chen, C., Xia, M., Wu, K., Chen, C., He, Q., Xue, G., Wang, W., He, Y. and Dong, Q. (2016) Interaction effects of $B D N F$ and COMT genes on resting-state brain activity and working memory. Front. Hum. Neurosci., 10, 540

34. Lewin, S. L., Utley, D. S., Cheng, E. T., Verity, A. N. and Terris, D. J. (1997) Simultaneous treatment with BDNF and CNTF after peripheral nerve transection and repair enhances rate of functional recovery compared with BDNF treatment alone. Laryngoscope, 107, 992-999

35. Kuleshov, M. V., Jones, M. R., Rouillard, A. D., Fernandez, N. F., Duan, Q., Wang, Z., Koplev, S., Jenkins, S. L., Jagodnik, K. M., Lachmann, A., et al. (2016) Enrichr: a comprehensive gene set enrichment analysis web server 2016 update. Nucleic Acids Res., 44, W90-W97

36. Namm, A., Arend, A. and Aunapuu, M. (2013) Pax proteins in embryogenesis and their role in nervous system development. Pap. Anthropol., 22, 133-142

37. Burrill, J. D., Moran, L., Goulding, M. D. and Saueressig, H. (1997) PAX2 is expressed in multiple spinal cord interneurons, including a population of $\mathrm{EN}^{+}{ }^{+}$interneurons that require PAX6 for their development. Development, 124, 4493-4503

38. Ziman, M. R., Rodger, J., Chen, P., Papadimitriou, J. M., Dunlop, S. A. and Beazley, L. D. (2001) Pax genes in development and maturation of the vertebrate visual system: implications for optic nerve regeneration. Histol. Histopathol., 16, 239-249

39. Raivich, G., Bohatschek, M., Da Costa, C., Iwata, O., Galiano, M., Hristova, M., Nateri, A. S., Makwana, M., Riera-Sans, L., Wolfer, D. P., et al. (2004) The AP-1 transcription factor c-Jun is required for efficient axonal regeneration. Neuron, 43, 57-67

40. Iorio, F., Bosotti, R., Scacheri, E., Belcastro, V., Mithbaokar, P., Ferriero, R., Murino, L., Tagliaferri, R., Brunetti-Pierri, N., Isacchi, A., et al. (2010) Discovery of drug mode of action and drug repositioning from transcriptional responses. Proc. Natl. Acad. Sci. USA, 107, 14621-14626

41. Sakaue, Y., Sanada, M., Sasaki, T., Kashiwagi, A. and Yasuda, H. (2003) Amelioration of retarded neurite outgrowth of dorsal root ganglion neurons by overexpression of $\mathrm{PKC} \delta$ in diabetic rats. Neuroreport, 14, 431-436

42. Duan, Q., Reid, S. P., Clark, N. R., Wang, Z., Fernandez, N. F., Rouillard, A. D., Readhead, B., Tritsch, S. R., Hodos, R., Hafner, M., et al. (2016) L1000CDS ${ }^{2}$ : LINCS L1000 characteristic direction signatures search engine. NPJ Syst. Biol. Appl., 2, 16015

43. Sun, F., Park, K. K., Belin, S., Wang, D., Lu, T., Chen, G., Zhang, K., Yeung, C., Feng, G., Yankner, B. A., et al. (2011) Sustained axon regeneration induced by co-deletion of PTEN and SOCS3. Nature, 480, 372-375

44. Agudelo, M., Gandhi, N., Saiyed, Z., Pichili, V., Thangavel, S., Khatavkar, P., Yndart-Arias, A. and Nair, M. (2011) Effects of alcohol on histone deacetylase 2 (HDAC2) and the neuroprotective role of trichostatin A (TSA). Alcohol. Clin. Exp. Res., 35, 1550 1556

45. Bolstad, B. M., Collin, F., Simpson, K. M., Irizarry, R. A. and Speed, T. P. (2004) Experimental design and low-level analysis of microarray data. Int. Rev. Neurobiol., 60, 25-58

46. Szklarczyk, D., Morris, J. H., Cook, H., Kuhn, M., Wyder, S., Simonovic, M., Santos, A., Doncheva, N. T., Roth, A., Bork, P., et al. (2017) The STRING database in 2017: quality-controlled protein-protein association networks, made broadly accessible. Nucleic Acids Res., 45, D362-D368

47. Breiman, L. I., Friedman, J. H., Olshen, R. A. and Stone, C. J. (1984) Classification and Regression Trees (CART). 1 Ed., Chapman and Hall/CRC

48. Chung, F. (2007) The heat kernel as the pagerank of a graph. Proc. Natl. Acad. Sci. USA, 104, 19735-19740

49. Vandin, F., Upfal, E. and Raphael, B. J. (2011) Algorithms for detecting significantly mutated pathways in cancer. J. Comput. Biol., 18, 507-522

50. Vandin, F., Clay, P., Upfal, E. and Raphael, B. J. (2012) Discovery of mutated subnetworks associated with clinical data in cancer. In Proceedings of the Pacific Symposium of Biocomputing 2012, pp. 55-66. World Scientific

51. Huang, W., Sherman, B. T. and Lempicki, R. A. (2009) Bioinformatics enrichment tools: paths toward the comprehensive 
functional analysis of large gene lists. Nucleic Acids Res., 37, 113

52. Huang, W., Sherman, B. T. and Lempicki, R. A. (2009) Systematic and integrative analysis of large gene lists using DAVID bioinformatics resources. Nat. Protoc., 4, 44-57

53. Mi, H., Huang, X., Muruganujan, A., Tang, H., Mills, C., Kang, D. and Thomas, P. D. (2017) PANTHER version 11: expanded annotation data from Gene Ontology and Reactome pathways, and data analysis tool enhancements. Nucleic Acids Res., 45, D183D189

54. Templeton, J. P. and Geisert, E. E. (2012) A practical approach to optic nerve crush in the mouse. Mol. Vis., 18, 2147-2152 